\title{
Hardwarearchitektur eines latenzoptimierten drahtlosen Kommunikationssystems für den industriellen Mobilfunk
}

\author{
Ludwig Karsthof ${ }^{1}$, Mingjie Hao ${ }^{1}$, Jochen Rust ${ }^{1}$, \\ Johannes Demel ${ }^{2}$, Carsten Bockelmann ${ }^{2}$, Armin Dekorsy ${ }^{2}$ \\ Stefan Meyering ${ }^{3}$, Jasper Siemons ${ }^{3}$, Ahmad Al Houry ${ }^{4}$, Fabian Mackenthun ${ }^{4}$, \\ Steffen Paul ${ }^{1}$ \\ ${ }^{1}$ Universität Bremen ITEM \\ \{karsthof, hao, rust, steffen.paul\}@item.uni-bremen.de \\ ${ }^{2}$ Universität Bremen ant \\ \{demel, bockelmann, dekorsy\}@ant.uni-bremen.de \\ ${ }^{3} \mathrm{IMST} \mathrm{GmbH}$ \\ \{stefan.meyering, siemons\}@imst.de \\ ${ }^{4}$ NXP Semiconductors Germany GmbH \\ \{ahmad.al.houry, fabian.mackenthun\}@nxp.com
}

\begin{abstract}
Zusammenfassung. Die immer stärker vernetzten und automatisierten Industrieanlagen erfordern zur Kommunikation der einzelnen Aktoren und Sensoren zunehmend den Einsatz von Funksystemen. Aufgrund der hohen Anforderungen an Stabilität der Funkverbindung durch Reflexion, Interferenz mit anderen Funksystemen und beweglichen Metallteilen können bestehende Standards in der Industrie nicht eingesetzt werden. Des Weiteren benötigen viele Anwendungen Echtzeitverarbeitung der gesendeten Nachrichten. Diese Arbeit stellt die im Rahmen des Forschungsprojekts HiFlecs implementierte innovative Hardware-Architektur für eine solche robuste Funklösung mit kurzer Latenz (1ms pro Link) vor. Das vollständig in Hardware entwickelte System weist eine geringe Paketfehlerwahrscheinlichkeit und Hardwarekomplexität auf und zeichnet sich durch geringe Leistungsaufnahme, Zuverlässigkeit und Echtzeitfähigkeit bezüglich der Anwendung aus.
\end{abstract}

\section{$1 \quad$ Einleitung}

Drahtlose Kommunikation im industriellen Umfeld gewinnt zunehmend an Bedeutung, da der Integrationsgrad an Sensoren und Aktoren in Fertigungsanlagen stetig ansteigt. Einige Industrieanwendungen benötigen für korrekte Funktionalität garantierte Latenzen von $1 \mathrm{~ms}$ [SLI $\left.{ }^{+} 17\right]$, um ihre Echtzeitbedingungen zu erfüllen. Aus diesen Spezifikationen ergibt sich der Bedarf an in der Industrie einsetzbaren Funksystemen. Derzeitige Funkstandards wie Zigbee, WLAN oder 
auch LTE erfüllen die Anforderungen der Industrie hinsichtlich der Robustheit oder Latenzzeit nicht. Beispielsweise für Fertigungsanlagen mit Echtzeit- und Sicherheitsanforderungen werden garantierte Latenzen unverzichtbar. Zu niedrigratige Update-Zyklen von Ist- und Soll-Positionen sich bewegender Elemente können bei instabiler Kommunikation ausfallen oder gar kritischen Schaden verursachen, in jedem Fall aber ist die maximale Bewegungsgeschwindigkeit und Leistungsfähigkeit der Anlage stark beeinträchtigt [PFBM16]. Bei den erwähnten Standards für Funksysteme handelt es sich um Funktechnologien, die hauptsächlich im Consumerbereich Anwendung finden, in denen keine Echtzeitanforderungen bestehen und demnach ein dynamisches Resource-Sharing das Timing und Scheduling übernimmt. Auch bestehen im Mobilfunk geringere Anforderungen der privaten Anwender an die Zuverlässigkeit der Übertragung (keine sicherheitskritische Übertragung). Trotz der kommenden 5G-Standardisierung sind gesonderte Konzepte zum industriellen Mobilfunk interessant, da sie Unabhängigkeit von Providern bieten und die voraussichtlich recht generische Peripherie um Industriestandards erweitern können. Versenden Kommunikationsteilnehmer in Mobilfunknetzen häufig Sprachsignale (Voice over IP) oder Bildmaterial, so sind üblicherweise versendete Messwerte oder Maschinenbefehle im industriellen Kontext erheblich kürzer. Im Forschungsprojekt HiFlecs werden von den Applikationen lediglich 256 Bit pro Uplink benötigt. Die 5G ITU-R Arbeitsgruppe der International Telecommunication Union (ITU) stellt diese Anforderungen ebenfalls an zukünftige Funktechnologien [IR15]. Das in dieser Arbeit vorgestellte Funksystem besitzt eine feste Pakettaktung nach dem Schema aus Abbildung 3. Das Scheduling der Kommunikation richtet sich nach dem Controller, welcher so getaktet ist, dass er jede Millisekunde eine Downlink-Broadcast (DL) Nachricht an alle Clients versendet. Die Länge des Downlink-Frames beträgt dabei $446,25 \mu \mathrm{s}$. Nach Umschalten der Clients von Empfangen auf Senden beginnt die Übertragung der Uplink-Daten, die zeitlich nacheinander versendet werden. Das Versenden eines OFDM-Symbols dauert 10,625 $\mu \mathrm{s}$. Die Payloadlängen betragen im DL als Broadcast 8192 Bit und im UL pro Nutzer 256 Bit. Das genutzte analoge Frontend überträgt hierbei gesteuert durch die hier entworfene Hardware der Basisbandsignalverarbeitung (BBSV) die Samples im OFDM-Spektrum mit 224 aktiven Trägern der Bandbreite $25,6 \mathrm{MHz}$ und verfügt über eine Fast-AttackAGC (Automatic Gain Control) zum schnellen Umschalten zwischen Senden und Empfangen mit kurzen Einschwingzeiten. Um die Authentizität und Integrität der Payload sicherzustellen wird im Security Modul ein Message Authentication Code verwendet. Zum Schutz der Vertraulichkeit werden die Daten zusätzlich verschlüsselt. Beides passiert durch latenzoptimierte Cipher-Implementierungen. Die entworfene Hardwarearchitektur umfasst die in Abbildung 2 aufgelisteten Blöcke, die in drei unterschiedliche Domänen der Signalverarbeitung aufgeteilt werden. Die SPI-Schnittstelle sowie das Security-Modul befinden sich in der Applikations-Domäne, die mit dem SPI-Takt von $40 \mathrm{MHz}$ getaktet ist. An den Schnittstellen der Taktdomänen befinden sich jeweils Dual-Port-RAM Speicherblöcke. Die Basisbandsignalverarbeitung arbeitet in ihrer eigenen Domäne und 
wird mit 50MHz getaktet. Das Streaming der Sendedaten an das Frontend findet in der Frontend-Domäne statt, hier muss mit $25,6 \mathrm{MHz}$ übertragen werden.



(a) Digilent Nexys-Video oben, RFFrontend unten

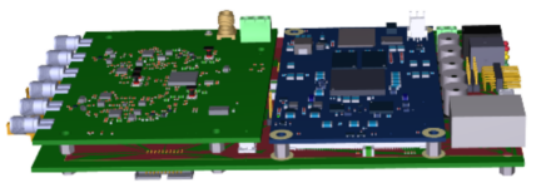

(b) CAD-Darstellung des RFFrontend Boards

Abb. 1. Darstellung der Boards des industriellen Funksystems, verbunden durch schnelle FMC-Verbindung.

Die entwickelte Hardware-Architektur wurde in VHDL implementiert und validiert. Die Ergebnisse der RTL-Synthese für FPGA-Anwendung, Latenz und Leistungsaufnahme sind Tabelle $2 \mathrm{zu}$ entnehmen, wobei ersichtlich wird, dass die Basisbandsignalverarbeitung schneller arbeitet als Daten verschickt werden können. Durch die festgelegte Übertragungsrate ergibt sich für die Algorithmik der digitalen Basisbandsignalverarbeitung eine Zeitreserve, die Optimierungspotenzial für zusätzliche Signalverarbeitung zur weiteren Stabilisierung der Funkübertragung bietet. In Abschnitt 2 wird zunächst näher auf das Einsatzgebiet des Funksystems eingegangen und ein Überblick über die Funktechnologie gegeben. Es folgt unter Kapitel 3.1 die Beschreibung des für die Industrie zwingend notwendige Sicherheits- und Authentifizierungsdienstes. Auch wird die Übertragungsqualität durch das Hinzufügen von Redundanz klassisch mittels Kanalkodierung erhöht, näheres hierzu folgt in Abschnitt 3.2. In den Kapiteln 3.5, 4.1 und 4.1 sind Details zum genutzten RF-Frontend, dessen Fast-Attack-AGC (Automatic Gain Control) und dem Vorgehen zur Synchronisierung sowie der Schätzung und Kompensierung der unterschiedlichen Taktfrequenzen zu finden. Ebenfalls geschätzt werden die Kanaleigenschaften, das Vorgehen hierzu ist beschrieben unter 4.2. Zusammenfassung und Bewertung folgen in Kapitel 5. 


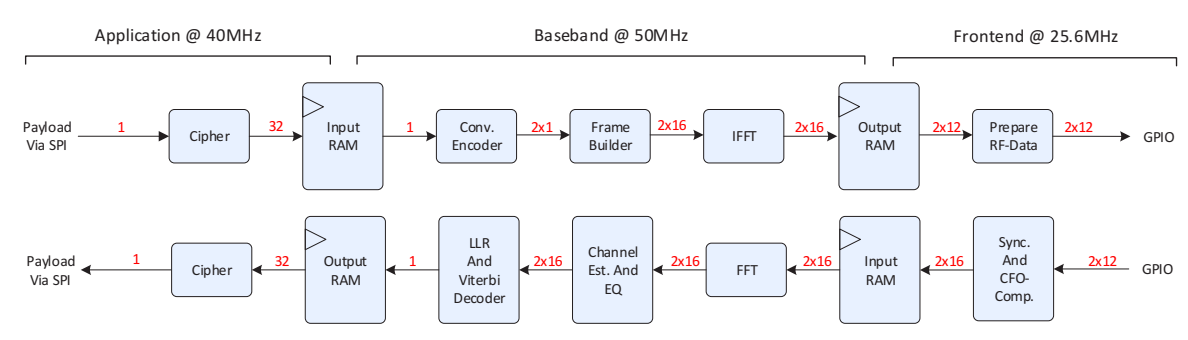

Abb. 2. Blockschaltbild der digitalen Signalverarbeitung von SPI-Master zur FMCSchnittstelle am RF-Frontend.

\section{Anforderungen}

Die Anforderungen an das System sind maßgeblich bestimmt durch die Umgebung in industriellen Fertigungshallen und der Objekte, die sich darin befinden. Mehrere Untersuchungen zu Kanälen in Industriehallen und anderen Anlagen wurden bereits durchgeführt [BFTM15][Cea17]. Aus diesen Untersuchungen gehen für Funkkanäle in Industrieanlagen hohe zeitliche Veränderlichkeit und Frequenzselektivität sowie Einflüsse aus Mehrwegeausbreitung hervor. Der umgesetzte Demonstrator besteht aus einem Förderband mit gesteuerten Transportschlitten und einem Roboter zur Fertigung. Die Schlitten weisen durch ihre Bewegung und die Drehung auf Wendestellen zeitliche Veränderung auf. Der Controller ist fest montiert und befindet sich im Schaltschrank. Die Anbindung der Industriekomponenten funktioniert über eine fest getaktete SPI-Schnittstelle.

\section{Systemdesign}

In diesem Kapitel wird das grundlegende Systemdesign näher beschrieben. Der Fokus liegt hierbei auf der genutzten Funktechnologie und der nachrichtentechnischen Konzepte, die Anwendung fanden, um die Anforderungen für den industriellen Mobilfunk zu erfüllen.

\subsection{Gewährleistung von Authentizität, Integrität und Vertraulichkeit}

Um Authentizität, Integrität und Vertraulichkeit der in Echtzeit übertragenen Nachrichten zu gewährleisten und gleichzeitig die Latenzanforderungen der Anwendungsfälle zu erfüllen, wurden geeignete Verschlüsselungsalgorithmen untersucht, echtzeitfähig in Hardware implementiert und für den Einsatz im HiFlecsSystem optimiert. Der Sender verschlüsselt die zu sendenden Daten und erzeugt ein Authentication-Tag, das an die verschlüsselten Daten angehängt wird. Der Empfänger entschlüsselt die Daten und prüft das Authentication-Tag, um sicher 
zu stellen, dass die Daten während der Übertragung nicht manipuliert wurden. Nur wenn das Authentication-Tag valide ist, dürfen die empfangenen Daten weitergereicht werden. Wird ein Fehler erkannt, muss das Paket verworfen und der Anlagenbetreiber darüber in Kenntnis gesetzt werden. Um auch zukünftig ein hohes Sicherheitslevel zu gewährleisten, werden die Echtzeitdaten in HiFlecs AES-verschlüsselt. Die Schlüssellänge beträgt 128 Bit. AES wird in HiFlecs im Counter-Modus (CTR) verwendet. Dieser sorgt dafür, dass zwei gleiche Eingangsblöcke unterschiedliche Ausgangsblöcke zur Folge haben, was u.a. eine Mustererkennung bei Eingangsdaten mit geringer Entropie verhindert. Damit folgt HiFlecs z.B. den aktuellen Empfehlungen des Bundesamtes für Sicherheit in der Informationstechnik (BSI) [BSI18]. Die optimierte AES-Implementierung wird ebenfalls für die Berechnung des Authentication-Tags genutzt. Hierzu wird das CMAC-Verfahren verwendet, welches ein 64-Bit Tag erzeugt, um die Authentizität der Daten zu gewährleisten.

\subsection{Kanalkodierung}

Die in dieser Arbeit genutzte Kanalkodierung fügt wie üblich Redundanz hinzu, um die Robustheit des Systems zu erhöhen. Hier kommt ein halbratiger Code zum Einsatz, sodass sich gegenüber unkodierter Payload eine halbierte Datenrate einstellt. Hierzu nimmt der Encoder pro Takt ein Bit Payload und gibt In-Phasen- und Quadraturkomponente (2 Bit) aus. Bei den genutzten Codes handelt es sich um einen einfachen Faltungscode mit der Einflusslänge 7 und den häufig genutzten Generatorpolynom-Oktetts $\left[133_{O C T}, 171_{O C T}\right]$. Es ergibt sich eine Anzahl von 6 Tail Bits, die den Datenstrom am Ausgang des Kodierers im Sendepfad verlängern. Der Dekodierer an der Empfangsseite ist ein Viterbi-Decoder. Die Soft-Bit-Eingänge des Viterbi wurden in der hier vorgestellten Version der Basisbandsignalverarbeitung nicht verwendet. Der Verzicht auf zusätzliche Performanz beim Dekodieren ermöglicht das Einsparen von Hardwarekomplexität und die einfachere Interpolation und Verwendung der Kanalkoeffizienten, da nun die Amplitudeninformation verworfen werden kann. Bei QPSK (Quadrature phase shift keying) liegt die Information in der Phase, empfangene Samples können durch einfache Addition oder Subtraktion entzerrt werden (siehe 4.2). Da durch die Faltung bei der Kodierung die Information von jedem einzelnen Bit über mehrere Unterträger verteilt wird, wurde zusätzlich ein Interleaving eingebaut. Dieses bewirkt, dass die Reihenfolge der zu übertragenden Bits geändert wird, sodass bei starker Beeinträchtigung mehrerer benachbarter Unterträger (zum Beispiel bei schmalbandiger Störung) beim Dekodieren nicht mehrere Fehlentscheidungen nacheinander getroffen werden und dadurch die Rekonstruktion fehlerhaft ist.

\subsection{Mehrträgerverfahren OFDM}

Beim vorliegenden System kommt ein Mehrträgerverfahren zum Einsatz, wobei orthogonales Frequenzmultiplexing (OFDM) genutzt wird. Da Störer oder Reflexionen bei Mehrwegeausbreitung häufig nur eine schmalbandige Störung der 
Funkübertragung verursachen, kann mittels Verteilen der Payload auf mehrere zueinander orthogonale Unterträger der Einfluss auf die gesamte Datenübertragung reduziert werden. Fehler in einzelnen Nutzdatenbits können wie bereits beschrieben durch das Hinzufügen von Redundanz mittels Kanalkodierung korrigiert werden. Die Übertragung findet hier auf 256 orthogonalen Unterträgern mit $100 \mathrm{kHz}$ Abstand statt. Hieraus ergibt sich die gesamte Breite des genutzten Spektrums zu 25,6MHz.

\subsection{Frame-Design}

Die Datenübertragung ist aufgeteilt in einzelne Zeitabschnitte (OFDM-Symbole) und die einzelnen Subträger. In Abbildung 3 ist die zeitliche Abfolge der Kom-

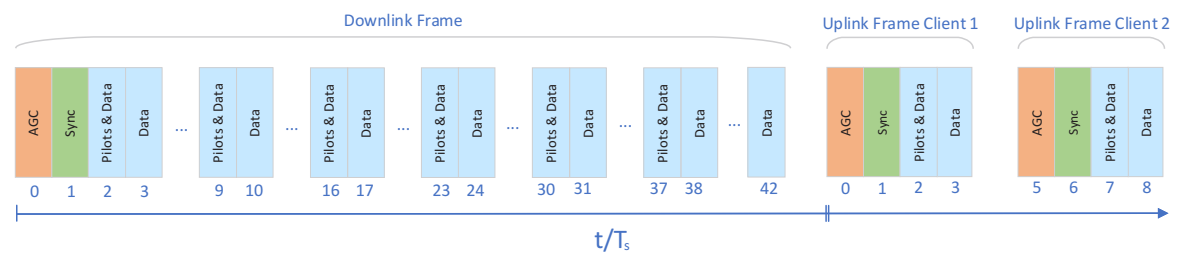

Abb. 3. Frame-Struktur des Setups. Zunächst wird eine Energiepräambel zum Einschwingen der AGC gesendet. Das zweite Symbol (grün) enthält die für die Synchronisierung entscheidende selbstähnliche Sequenz, anschließend werden die Daten gesendet. Gleichmäßig auf die Daten-OFDM-Symbole verteilt werden Pilot versendet, die der Entzerrung des Kanals dienen. Die Symboldauer $T_{S}$ beträgt $10.625 \mu \mathrm{s}$.

munikation zu sehen. Auf der linken Seite der Abbildung beginnend wird im Downlink zunächst eine Energie-Präambel gesendet. Dies dient dem Einschwingen der automatischen Verstärkungsregelung (Automatic Gain Control, $A G C$ ). Da die Präambel dieselbe mittlere Energie aufweist wie während des regulären Betriebs festzustellen ist, bleibt die Verstärkung bei Übertragung der Nutzdaten konstant. Das Nachregeln und Einschwingen der AGC während der Übertragung von Nutzbits oder der Synchronisierungspräambel könnte Fehler beim Finden des Startpunkts der Übertragung im Empfänger, oder sogar Fehler in den Nutzdatenbits erzeugen. Das zweite übertragene OFDM-Symbol beinhaltet die Synchronisierungspräambel, wie in Abschnitt 4.1 beschrieben. Nachdem die beiden Präambeln gesendet wurden, werden Daten versandt, wobei in festem Abstand von sieben Symbolen Piloten gesendet werden, die der Kanalschätzung dienen. Untersuchungen zur Frequenzselektivität und zeitlichen Varianz von Kanälen in industriellen Umgebungen [BFTM15] ergaben eine durch Reflexion an Metallteilen (demnach Mehrwegeausbreitung) und sich bewegende Funkteilnehmer hohe Veränderlichkeit des Funkkanals. In dieser Implementierung wurde daher jeder vierte Unterträger mit Piloten belegt, woraus direkt Kanalkoeffizienten berechnet werden. Zwischen den Kanalkoeffizienten wird für die übrigen Unterträger 
linear interpoliert. Dies wirkt der Frequenzselektivität entgegen, wohingegen für die Zeitvarianz häufig geschätzt werden muss. Im Downlink passiert dies alle $74,375 \mu s$, im Uplink wurde für jeden Client ein OFDM-Symbol mit gleichem Unterträgerabstand bereitgestellt, dessen Piloten für zwei OFDM-Symbole (also $21,25 \mu \mathrm{s}$ ) Gültigkeit besitzen. Nutzertrennung im Uplink passiert in diesem Design im Zeitbereich, wobei getrennt durch Pausen der Länge eines OFDMSymbols den Clients eigene Zeitschlitze zugewiesen sind (Time Division Multiple Access, TDMA). Die Empfangsdaten an der Antenne eines Setups mit zwei im Uplink sendenden Clients ist in Abbildung 4 dargestellt. Zu Beginn der Übertragung ist das Frontend noch nicht auf Empfangen geschaltet. Dies wird es, sobald in Abbildung 4 circa bei Sample 4000 der Uplink beginnt. Zunächst sendet das Frontend über den FMC-Connektor nur das empfangene Rauschen, bis Client eins kurze Zeit später beginnt, hohe Aussteuerung aufzuweisen. Dies indiziert die Zeit, in der die AGC sehr hohe oder sehr niedrige Werte nahe full-scale an die Signalverarbeitung sendet, da die Verstärkung zunächst sehr hoch eingestellt war und sich nun einschwingt. Mit einer Pause von etwa einem OFDM-Symbol folgt dann die Übertragung des Clients zwei. Zwischen den einzelnen OFDMSymbolen wurde ein Cyclic Prefix eingefügt, welches als Guard Interval Interferenz zwischen den Symbolen verhindert. Hierfür werden die letzten 16 Samples jedes OFDM-Symbols an den Beginn des OFDM-Symbols kopiert.
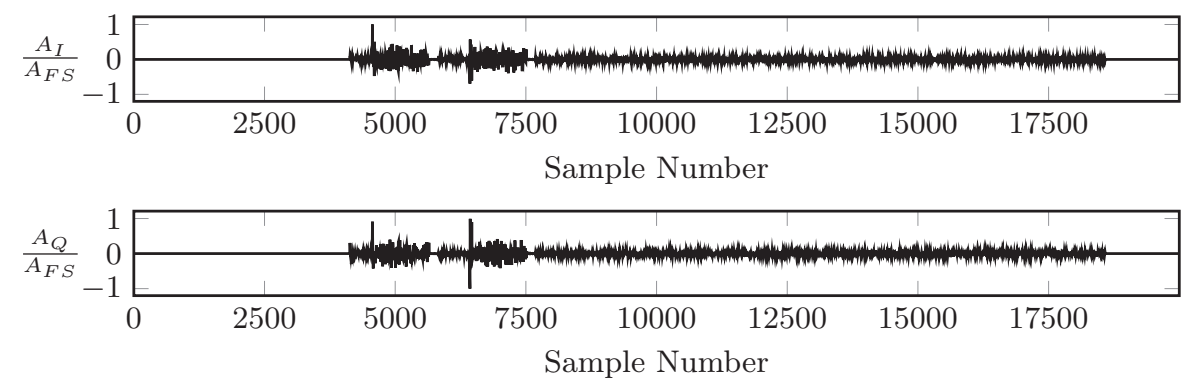

Abb. 4. Empfangsdaten von der Antenne des System-Controllers mit $A_{I}$ als InPhasenkomponente, $A_{Q}$ Quadraturkomponente normiert auf die maximale Aussteuerung $A_{F S}$.

\subsection{RF-Frontend und AGC}

Frontend-Hardware Ein wesentlicher Bestandteil des neu entwickelten Funksystems ist das Frontend. Das HF-Frontend enthält eine Vorfilterung und Verstärkung der analogen Signale, einen Transceiver mit einer nachgeschalteten schnellen Digitalverarbeitung für zusätzliche Filterungen und Ratenanpassungen sowie Debug- und Kontrollschnittstellen. Die Filter sind auf das Frequenzband $5,725 \mathrm{GHz}$ bis $5,875 \mathrm{GHz}$ ausgelegt und das Frontend kann mit den Signalverstärkern eine maximale Sendeleistung von $17 \mathrm{dBm}$ erreichen. Das Mischen 
ins Basisband, die schnelle automatische Pegelanpassung AGC und die AnalogDigital-Wandlung erfolgen im Transceiver (AD9361 Chip von Analog Devices). Als Schnittstelle zum Basisband-Board dient ein FMC-Connector (FPGA Mezzanine Card). Die Kernparameter sind in Tabelle $1 \mathrm{zu}$ sehen.

Tabelle 1. Wichtige HF-Frontend Parameter im Überblick

\begin{tabular}{ll}
\hline Parameter & Range \\
\hline Frequenzband & $5,725 \mathrm{GHz}$ bis $5.875 \mathrm{GHz}$ \\
HF Kanalbandbreite & Maximal 25MHz, Startwert 10MHz \\
Betriebsarten & Einkanaliger TDD, Zweikanaliger TDD, FDD \\
Sendeleistung & Maximal $17 \mathrm{dBm}$ (optional max. 26dBm) \\
IQ Abtastrate & Maximal $50 \mathrm{MHz}$ \\
\hline
\end{tabular}

Schnelle automatische Pegelanpassung Um die Echtzeitbedingung für Industrieanwendungen zu erfüllen, werden sehr kurze Reaktionszeiten benötigt. Daher wurde ein schneller AGC-Algorithmus entwickelt und untersucht, der die Einschwingzeit des Funksystems minimiert. Ausschlaggebend für die kurze Reaktionszeit der AGC ist, dass der AGC-Algorithmus ohne Interaktion mit den Basisbandkomponenten auskommt, um die Pegelanpassung durchzuführen. Der Algorithmus besteht aus zwei Phasen, einer sehr schnellen Grobjustierung zur situationsangepassten Reduktion des Pegels und einer Feinjustierung zur Verbesserung der Genauigkeit des Einstellwertes. Bei der Grobjustierung werden Spitzenwert-Detektoren verwendet, die direkt bei Eintreffen eines Pakets mögliche Signalpegelüberschreitungen melden und eine grobe Einstellung des Verstärkungsfaktors in kürzester Zeit ermöglichen. Die Feinjustierung in der zweiten Phase basiert auf Messungen der momentanen Eingangsleistung.

\section{Hardwarearchitektur}

Im folgenden Kapitel wird die Hardwarearchitektur des Funksystems näher beschrieben. Fokus liegt hier auf der digitalen Basisbandsignalverarbeitung. Dieser Abschnitt ist unterteilt in drei Domänen, die nach Abbildung 2 durch die jeweilige Taktdomäne bezeichnet sind. Bei dieser Arbeit war die Taktfrequenz der SPI-Schnittstelle durch die Applikation vorgegeben und wurde daher auf $40 \mathrm{MHz}$ festgelegt. Durch das Senden und Empfangen von Daten auf 256 Unterträgern mit $100 \mathrm{kHz}$ Abstand ist auch die Schnittstelle zum Frontend-Board auf $25,6 \mathrm{MHz}$ festgelegt. Alle Berechnungen im Basisband werden mit $50 \mathrm{MHz}$ getaktet, da dies einen guten Kompromiss zwischen Hardwarekomplexität und Echtzeitanforderung darstellt. Die Elemente der einzelnen Domänen werden im Folgenden vorgestellt. 


\subsection{Frontend-Domäne}

Die Frontend-Domäne beinhaltet alle Module, die das Sende- oder Empfangssignal im Zeitbereich verarbeiten. Im Zeitbereich wird die Frame-Synchronisierung durchgeführt und der Frequenzversatz der Oszillatoren verschiedener Funksystemteilnehmer ausgeglichen (Carrier Frequency Offset, CFO).

Synchronisierung Die hier genutzte Framedetektion basiert auf dem in [SC97] vorgestellten Verfahren zur Synchronisierung. Dazu wird eine Zadoff-Chu-Sequenz der Länge eines OFDM-Symbols verwendet. Nach Definition besitzt diese eine konstante Amplitude und eine kleine Autokorrelation. Im vorliegenden System unterscheidet sie sich in Uplink und Downlink. Die Sequenzen sind vorprozessiert und damit auch im Sendepfad der iFFT nicht zugeführt. Dies optimiert die Latenz des Gesamtsystems um 256 Takte, da die Präambel gesendet werden kann, während die ersten Datensymbole noch verarbeitet werden. Im Zeitbereich betrachtet besitzt die Präambel zwei identische Teile (Zadoff-ChuSequenzen) der Länge 128. Empfängt ein Teilnehmer eine solche Sequenz, so detektiert der hier implementierte Synchronisierungsalgorithmus mittels Autokorrelationsfunktion (ACF)

$$
X_{A C F}=\sum_{n=0}^{127} x_{n} \cdot x_{n+128}^{*}
$$

und Kreuzkorrelation (Cross Correlation Function, XCF) die Präambel. Bei der ACF handelt es sich um eine Time lagged ACF, die zu jedem diskreten Zeitpunkt eine Ähnlichkeit der empfangenen Samples mit den vor 128 Takten verarbeiteten Samples ergibt. Hierbei ergibt sich ein Plateau von der Breite einiger Samples, die zur Framedetektion nicht ausreichend sind. Daher wird diese nach [AKE08] quadriert und mit der Kreuzkorrelation des Empfangssignals mit der bekannten Synchronisierungspräambel multipliziert. Da die bei der $X C F$ entstandenen Spitzen geringerer Aussteuerung abseits des eigentlichen Synchronisierungszeitpunkts nun mit dem Quadrat der $A C F$ multipliziert werden, ergibt sich nun ein schmaler Puls hoher Aussteuerung, der den Beginn des Frames indiziert. In Abbildung 5 ist von einem über die Luft übertragenen Downlink das Ergebnis der eben genannten Funktionen im ersten OFDM-Symbol dargestellt.

Wird nun im Downlink eine Präambel detektiert, beginnt die Schätzung des Frequenzoffsets im Zeitbereich. Das Vorgehen hierzu ist in Abschnitt 4.1 beschrieben. Der Hardware-Controller puffert im RAM Empfangswerte und kann nach detektiertem Framebeginn und berechneter $C F O$ diese an die $C F O$-Kompensierung weitergeben.

CFO-Schätzung und -kompensierung In jedem Funksystem werden Taktgeber verwendet, die aufgrund von Fertigungstoleranzen von ihrer vorgegebenen Taktfrequenz abweichen. Da es sich beim Sender und Empfänger einer Nachricht um unterschiedliche Systeme handelt, entsteht beim Hochmischen im einen und 

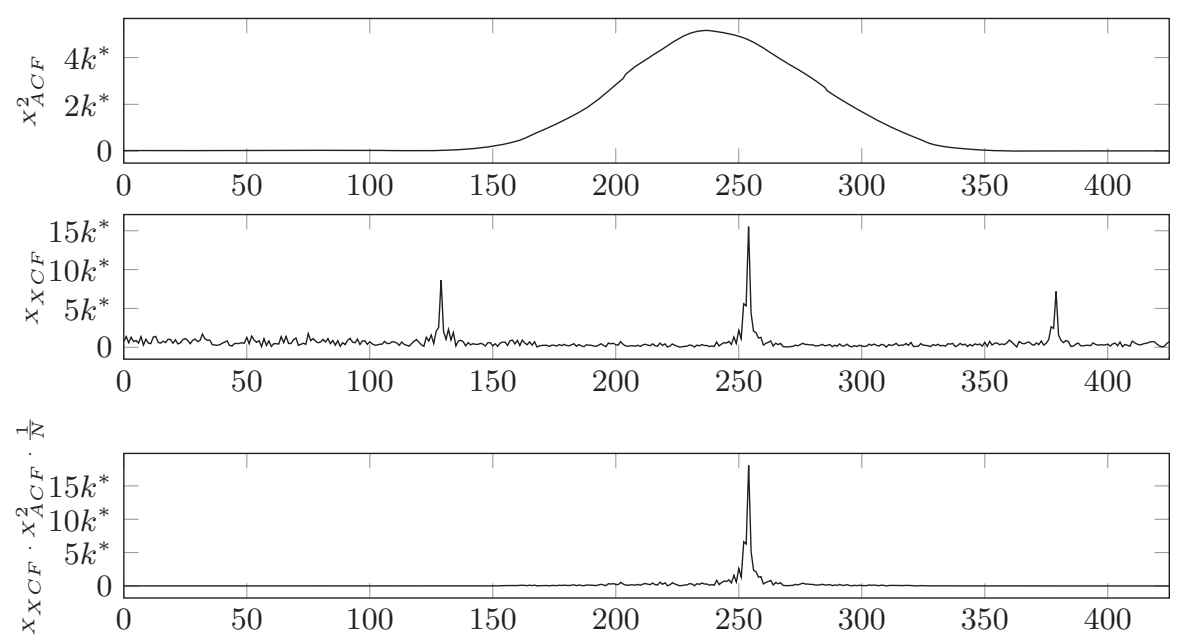

Abb. 5. Quadrat der Autokorrelation und Kreuzkorrelation werden multipliziert und mit N normalisiert. Daten gemessen während des Funkbetriebs. ${ }^{*}$ Fixpointdarstellung mit $2^{16}$ multipliziert.

Heruntermischen im anderen ein Frequenzversatz der Unterträger ( $C F O$ ). Dieser kann bei Addition der Versätze Fehler hervorrufen und muss kompensiert werden. Der $C F O$ lässt sich im Zeitbereich durch unterschiedliche Sampledauer der Teilnehmer veranschaulichen, die eine Drehung der Empfangswerte gegenüber den eigentlich gesendeten Samples bewirkt. In Abschnitt 4.1 wurde zur Synchronisierung der Frames bereits die implementierte Autokorrelation erklärt, deren Ergebnisfunktion die Ähnlichkeit mit sich selbst vor 128 Takten beschreibt.

$$
\Phi_{A C F}=\arctan \left(\frac{\Im\left\{X_{A C F}\right\}}{\Re\left\{X_{A C F}\right\}}\right)
$$

Der Winkel $\Phi_{A C F}$ dieser Autokorrelation beschreibt nun die Drehung der Samples gegenüber der Drehung von vor 128 Takten, wodurch der CFO bestimmt ist. Der Winkel der Autokorrelation wird mithilfe einer linearen Approxmiationsfunktion angenähert und weitergegeben an die Module zur CFO-Kompensation,

welche die Drehung im Zeitbereich durch Multiplikation mit der Drehmatrix kompensiert:

$$
\left(\begin{array}{l}
y_{R}^{\prime} \\
y_{I}^{\prime}
\end{array}\right)=\left(\begin{array}{cc}
\cos \left(\Phi_{A C F}\right) & -\sin \left(\Phi_{A C F}\right) \\
\sin \left(\Phi_{A C F}\right) & \cos \left(\Phi_{A C F}\right)
\end{array}\right) \cdot\left(\begin{array}{l}
y_{R} \\
y_{I}
\end{array}\right)
$$

Die Winkelfunktionen $\sin \Phi_{A C F}$. und $\cos \Phi_{A C F}$ werden ebenfalls linear approximiert. Ein restlicher $C F O$-Effekt, der durch diese Approximationen verbleibt, kann durch die Kanalschätzung entzerrt werden. 


\subsection{Basisband-Domäne}

Die Basisband-Domäne beinhaltet die gesamte digitale Signalverarbeitung die im Basisband stattfindet. Dies betrifft die Hin- und Rücktransformation der Daten von Frequenzbereich zu Zeitbereich, Schätzung der Kanäle, Entzerrung, (De-)Kodierung und Frame-Design.

Schnelle Fourier-Transformation Nach Synchronisierung und CFO-Kompensierung wird das empfangene Signal vom Zeit- in den Frequenzbereich transformiert. Dies funktioniert mithilfe der diskreten Fourier-Transformation, in Hardware implementiert als Fast Fourier Transform (FFT). Bei der hier implementierten FFT handelt es sich um eine sequenzielle Radix- $2^{2}$ SDF Implementierung. Die Hardware-Architektur wurde durch uns bereits in $\left[\mathrm{KHR}^{+} 16\right]$ genau beschrieben. An dieser Stelle sollen nur die wichtigsten Eigenschaften zusammengefasst werden. Sowohl für den Uplink als auch für den Downlink werden 256 Subträger belegt und damit auch eine 256-Punkt-FFT umgesetzt. Die Hardwareimplementierung wird sowohl für die Vorwärts- als auch für die Rückwärts-FFT verwendet, da sie sich nur unterscheiden durch die Normierung, die Input-Samples und die Drehrichtung (Vorzeichen Exponent der Eulerschen Zahl). Hier kann durch eingangs- und ausgangsseitiges Vertauschen der Real- und Imaginärteile der Samples die Vor- und Rücktransformation erreicht werden. Die FFT besitzt acht Butterfly-Strukturen, die getrennt sind durch Pipieline-Register. Diese sorgen für eine Verkürzung des kritischen Pfads und damit zu einer höheren möglichen Taktfrequenz, mit der das System betrieben werden kann. TwiddleFaktoren werden in ROMs abgelegt und ausgelesen. Durch die Butterfly-Struktur und die Pipeline-Register ergibt sich eine Latenz von 256+8 Takten bei einer Genauigkeit von 16 Bit . Intern wird nach den einzelnen Operationen der Datenpfad verbreitert, erst beim Ergebnis wird das Ergebnis wieder auf 16 Bit reduziert, sodass sich Quantisierungseffekte durch endliche Genauigkeit nach Multiplikationen und Additionen nicht innerhalb der FFT verstärken.

Kanalschätzung Kanalschätzung und -entzerrung werden in diesem Kapitel näher beschrieben. Ausgangspunkt ist der Frame, der in diesem Punkt im Design bereits durch die schnelle Fourier-Transformation in den Frequenzbereich zurück transformiert wurde. Bei der Kanalschätzung wird üblicherweise eine Kanalmatrix, bestehend aus Kanalkoeffizienten berechnet, die durch Multiplikation mit zugehörigen Empfangs-Samples den entzerrten Wert, idealerweise den Sendewert, ergeben. Bedingt durch das Pilotendesign wurde aufgrund der simplen Interpolation zwischen geschätzten Unterträgern lediglich die Phaseninformation der IQ-Samples verwendet. Dadurch ergibt sich für die Schätzung eine einfache lineare Interpolation zwischen den Kanalkoeffizienten der Subträger, und die Kanalkoeffizienten werden dargestellt durch einfache Korrekturwerte, die durch additive Verrechnung mit Empfangswerten die Samples entzerren können. In Abbildung 6 ist die Funktionsweise der Kanalschätzung als Blockschaltbild dargestellt. Die IQ-Samples des Ausgangs aus der Fourier-Transformation werden 


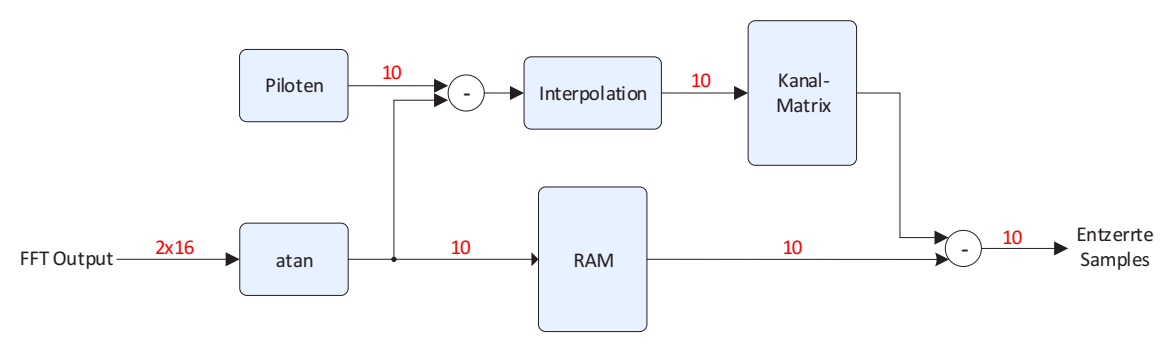

Abb. 6. Blockschaltbild Kanalschätzung und -entzerrung

zunächst mit einer linearen Approximation des Arkustangens nach $\left[\mathrm{KHR}^{+}{ }^{16}\right]$ angenähert und deren Phase bestimmt. Da sich Payload zwischen den PilotSamples befindet, müssen zur Entzerrung dieser Samples erst die Kanalkoeffizienten bekannt sein. Da eine Entzerrung ohne gültige Kanalkoeffizienten nicht möglich ist, werden die zu entzerrenden Werte in einem Speicher (RAM-Block in Abbildung 6) für die Dauer eines OFDM-Symbols (256 Takte) zwischengespeichert und später entzerrt. Da der Kanal über einige zig Mikrosekunden als konstant angenommen wird, und Flat Fading vorausgesetzt wird, beinhalten nur manche Symbole Piloten (vgl. Abbildung 3) und diese belegen nicht alle Unterträger innerhalb eines Pilotensymbols. Zwischen ihnen wird daher interpoliert, wie in Abbildung 6 gezeigt.

\subsection{Applikationsdomäne}

Das implementierte System verfügt über eine Anbindung an Applikationen via Serial Peripheral Interface (SPI). Die SPI-Implementierung ist hierbei in einer eigenen Taktdomäne umgesetzt, da die angebundene Applikation als Randbedingung einen eigenen Takt verlangt. Die angeschlossenen Aktoren der Industrieanlage sind aufgrund der festen Rasterung des UL/DL SPI-Slaves, auf dem Funksystem ist ein SPI-Master umgesetzt. Ebenfalls in der Applikationsdomäne ist das unter Abschnitt 3.1 beschriebene Security-Modul instanziert. Da durch die Struktur der Sendedaten am Ausgang des Cipher-Moduls keine Rückschlüsse auf die eigentlichen Payload-Bits möglich sind, verbessert diese Umsetzung neben der Sicherheit im Funksystem auch die Übertragungseigenschaften und stellt Payload-Unabhängigkeit her, da stets eine Gleichverteilung der Symbole auch bei konstanten Sendedaten vorliegt. Dies verbessert (verringert) die Peakto-average-power ratio (PAPR), die zu Fehlern führen kann, falls die AGC (vgl. 3.5) während der Übertragung von Daten nachregelt. Da der gesamte Wertebereich des Empfangssignals aufgelöst werden muss, um Daten rekonstruieren zu können, bewirkt die Gleichverteilung und Umsortierung der Sendedaten eine höhere Übertragungsqualität. Die Cipher nimmt nach Entzerrung der Samples die Daten und entschlüsselt diese, wobei entschieden wird, ob der übertragene MAC-Header gültig ist. Im Uplink wird dies für jede zeitlich getrennte Client- 
Nachricht einzeln ausgewertet und den Quality-of-Service-Bits (QoS) hinzugefügt, durch die die Applikation die Validität von Nachrichten überprüfen kann. Der Übertragung der Payload via SPI wird nun noch eine Checksumme (CRC) hinzugefügt. Die SPI-Kommunikation des Systems ist in Abbildung 7 dargestellt. Für die Checksumme wurde der Algorithmus CRC-32/BZIP2 mit dem Polynom 0x04C11DB7 verwendet.

Downlink (DL) Funksystem



Uplink (UL) Funksystem



Abb. 7. Struktur der SPI-Kommunikation des industriellen Funksystems mit der Applikation

\section{$5 \quad$ Auswertung der Implementierung}

In dieser Arbeit wurde die Hardwarearchitektur eines in der Industrie einsetzbaren Funksystems vorgestellt. Es verfügt über eine Zykluszeit von 1ms und wurde getestet. Als Demonstrator wurde das Funksystem mit einer SPI-Anbindung in Fertigungsanlagen eingebaut und dort in Betrieb genommen. Im Folgenden sollen Kernparameter und Eigenschaften mit Fokus auf der digitalen Basisbandsignalverarbeitung ausgewertet werden.

\subsection{Hardwarekomplexität}

Eine Übersicht zur Implementierung auf einem Xilinx Artix-7 FPGA ist in Tabelle 2 zu sehen. Besonders auffällig sind hierbei die hohe Komplexität der Kreuz- 
Tabelle 2. Hardwarekomplexität des Controllers (Clients), synthetisiert @ $50 \mathrm{MHz}$. Die Latenz gibt die Zeitspanne vom Startzeitpunkt der Verarbeitung bis zu den ersten gültigen Ergebnissen markiert. Die kumulierten Ergebnisse ${ }^{*}$ spiegeln die Verarbeitungszeiten der gesamten digitalen Basisbandsignalverarbeitung mit Speicherzugriffen wieder.

\begin{tabular}{l|c|c|c|c|c}
\hline Modul Name & Slices LUTs & Slices Reg. & BRAM & DSP & Takte Latenz \\
\hline Cipher (Encryption) & $1086(983)$ & $1243(1198)$ & 0 & 0 & $1945(75)$ \\
Convolutional Enco- & $112(108)$ & 357 & 0 & 0 & 2 \\
der & $2001(2050)$ & 10375 & 2 & 12 & 265 \\
IFFT & $200(202)$ & 139 & 1 & 0 & 5 \\
Frame builder & $54(49)$ & 32 & 2.5 & 12 & - \\
Weitere & $\mathbf{3 4 5 3 ( 3 3 9 2 )}$ & $\mathbf{1 2 1 4 6}(\mathbf{1 2 1 0 1})$ & $\mathbf{5 . 5}$ & $\mathbf{1 2}$ & $\mathbf{8 9 1 6}$ \\
TX total: & 28527 & 14741 & 0 & 552 & 274 \\
\hline Synchronisierung & 5855 & 3575 & 0 & 4 & 1 \\
CFO Kompensierung & 1940 & 9811 & 2 & 12 & 265 \\
FFT & $1338(1333)$ & 1298 & 0 & 0.5 & 14 \\
Kanalschätzung & $40(43)$ & 290 & 0.5 & 0 & 244 \\
Entzerrung & $3344(3330)$ & $1540(1531)$ & 0 & 0 & $161(226)$ \\
Viterbi Decoder & $1764(1915)$ & $1553(1610)$ & 0 & 0 & $1949(79)$ \\
Cipher (Decryption) & $446(471)$ & 1356 & 0.5 & 0 & - \\
Weitere & $\mathbf{4 3 2 4 4 ( 4 3 4 1 4 )}$ & $\mathbf{3 4 1 6 4 ( 3 4 2 1 2 )}$ & $\mathbf{3}$ & $\mathbf{4 6}$ & $\mathbf{1 1 2 4 4}(\mathbf{1 3 9 5 9 )}$ \\
RX total & \multicolumn{4}{|c|}{}
\end{tabular}

korrelation (in Synchronisierung enthalten) und der Kanalschätzung. Die Kanalschätzung benötigt durch die gespeicherten Pilotensymbole viele LUTs, hingegen sind für die Kreuzkorrelation besonders viele Multiplizierer (DSP-Slices) notwendig.

\section{Förderung der Forschung}

Diese Arbeit wurde im Rahmen des Forschungsprojekts HiFlecs(Referenznummer 16KIS0271) gefördert vom deutschen Bundesministerium für Bildung und Forschung (BMBF).

\section{Literatur}

[AKE08] A. B. Awoseyila, C. Kasparis und B. G. Evans. Improved preamble-aided timing estimation for OFDM systems. IEEE Communications Letters, 12(11):825-827, November 2008.

[BFTM15] D. Block, N. H. Fliedner, D. Toews und U. Meier. Wireless channel measurement data sets for reproducible performance evaluation in industrial environments. In 2015 IEEE 20th Conference on Emerging Technologies Factory Automation (ETFA), Seiten 1-4, Sept 2015. 
[BSI18] BSI. BSI - Technische Richtlinie. https://www.bsi.bund.de/SharedDocs/

Downloads/DE/BSI/Publikationen/TechnischeRichtlinien/TR02102/

BSI-TR-02102 .pdf ; jsessionid=42D20DAF775FB0C2476C6AC5CC9C0CB6 . 2_ cid369?__blob=publicationFile\&v=8, 2018.

[Cea17] R. Candell und M. Kashef et al. Industrial wireless systems: Radio propagation measurements, National institute of Standards and Technology, Gaithersburg, MD, Tech.Rep., Available: http://nvlpubs.nist.gov/nistpubs/TechnicalNotes/NIST.TN.1951.pdf. 2017.

[IR15] ITU-R. Framework and Overall Objectives of the Future Development of IMT for 2020 and Beyond. ITU, Feb. 2015.

$\left[\mathrm{KHR}^{+} 16\right]$ L. Karsthof, M. Hao, J. Rust, D. Block, U. Meier und S. Paul. Dynamically reconfigurable real-time hardware architecture for channel utilisation analysis in industrial wireless communication. In 2016 IEEE Nordic Circuits and Systems Conference (NORCAS), Seiten 1-6, Nov 2016.

[PFBM16] K. I. Pedersen, F.Frederiksen, G. Berardinelli und P. E. Mogensen. The Coverage-Latency-Capacity Dilemma for TDD Wide Area Ooperation and Related 5G Solutions. In Veh. Technol. Conf. (VTC Spring). IEEE, Mai 2016.

[SC97] T. M. Schmidl und D. C. Cox. Robust frequency and timing synchronization for OFDM. IEEE Transactions on Communications, 45(12):1613-1621, Dec 1997.

[SLI $\left.{ }^{+} 17\right]$ H. Shariatmadari, Z. Li, S. Iraji, M. A. Uusitalo und R. Jäntti. Control channel enhancements for ultra-reliable low-latency communications. In 2017 IEEE International Conference on Communications Workshops (ICC Workshops), Seiten 504-509, May 2017.

Open Access Dieses Kapitel wird unter der Creative Commons Namensnennung 4.0 International Lizenz (http://creativecommons.org/licenses/by/4.0/deed.de) veröffentlicht, welche die Nutzung, Vervielfältigung, Bearbeitung, Verbreitung und Wiedergabe in jeglichem Medium und Format erlaubt, sofern Sie den/die ursprünglichen Autor(en) und die Quelle ordnungsgemäß nennen, einen Link zur Creative Commons Lizenz beifügen und angeben, ob Änderungen vorgenommen wurden.

Die in diesem Kapitel enthaltenen Bilder und sonstiges Drittmaterial unterliegen ebenfalls der genannten Creative Commons Lizenz, sofern sich aus der Abbildungslegende nichts anderes ergibt. Sofern das betreffende Material nicht unter der genannten Creative Commons Lizenz steht und die betreffende Handlung nicht nach gesetzlichen Vorschriften erlaubt ist, ist für die oben aufgeführten Weiterverwendungen des Materials die Einwilligung des jeweiligen Rechteinhabers einzuholen. 